Results Bulk flow sorted cell populations (CD45, epithelial) from kidney samples can separate LN from controls based on gene expression. IFN stimulated genes were differentially expressed in renal CD45 LN cells. Analysis of single cells sorted from $4 \mathrm{LN}$ kidney biopsies revealed major differences in infiltrates composition, with 2 samples demonstrating a high percentage of $\mathrm{B}$ cells (average of $18 \%$ compared to no B cells in the other 2 samples) and CD4 T cells (18\% vs 8\%), and low percentage of CD8 T cells (9\% vs 23\%). A high transcriptomic lupus interferon signature was detected in urine CD45 cells. Distinct infiltrates and distinct expression profiles were detected across patients.

Conclusions The PEARL-Phase 0 project shows the feasibility of single cell isolation and transcriptomic analysis from LN kidney and urine. Analyses at a bigger scale in the two next phases of the project will accelerate discovery of new therapeutic targets and identification of biomarkers to guide therapeutic decisions in lupus nephritis and integrate the treatment effect.

\section{THE EXPRESSION OF SIGLEC-10 IS ELEVATED ON B LYMPHOCYTES AND ASSOCIATED WITH DISEASE ACTIVITY IN PATIENTS WITH SYSTEMIC LUPUS ERYTHEMATOSUS}

J Bo miao*, P Dan, L Xiaohong, H Zhiming, Z Li, Y Zijing, H Lan. xi'an jiaotong university, First Affiliated Hospital, xi'an, China

\subsection{6/lupus-2017-000215.265}

Background and aims Siglec-10 (sialic acid-binding immunoglobulin-like lectins-10) is a member of Siglecs family and known to be expressed on B cells to regulate immune tolerance. This study aimed to investigate the expression of Siglec-10 on B cells of SLE and evaluate anti-inflammatory and immunosuppressive drugs impact on the expression of Siglec-10 on B cells in vitro.

Methods Peripheral blood mononuclear cells (PBMC) were obtained from patients with SLE $(n=57)$ and healthy donors $(n=30)$. Flow cytometry was performed to examine the expression of Siglec-10 on B cells. The potential association of these measures with the values of SLE disease activity index (SLEDAI) score was also analysed. In vitro cultivation of PBMC with Dexamethasone sodium phosphate and chloroquine phosphate, the expression of Siglec-10 on B cells were detected by flow cytometry after 16 hour.

Results The expression of Siglec-10 on B cells was significantly increased in SLE patients compared with normal controls $(\mathrm{p}<0.001)$. Moreover, it was positively correlated with SLEDAI $(O R=0.277, p<0.05)$ and elevated in non-treatment patient than patients after anti-rheumatic treatment $(\mathrm{p}<0.05)$. In vitro assay, Dexamethasone sodium phosphate and chloroquine phosphate could significantly decreased the expression of Siglec- 10 on B cells respectively $(\mathrm{p}<0.05)$.

Conclusions The results from this study imply that Siglec-10 may play a potential role in progression and pathogenesis of SLE, which may represent a therapeutic target in SLE patients.

\section{HUMAN AUTOANTIBODIES FROM CHILDREN WITH MOVEMENT AND PSYCHIATRIC DISORDERS TARGET THE EXTRACELLULAR N-TERMINUS OF DOPAMINE-2 RECEPTOR}

N Sinmaz, F Tea, D Pilli, A Zou, T Nguyen, V Merheb, S Ramanathan, R Dale, F Brilot*. The Children's Hospital at Westmead- Kids Research Institute- University of Sydney, Brain Autoimmunity Group- Institute for Neuroscience and Muscle Research, Westmead, Australia

\subsection{6/lupus-2017-000215.266}

Background and aims We recently identified dopamine-2 receptor (D2R) autoantibodies in children with autoimmune movement and psychiatric disorders. This supported the hypothesis that a subgroup of patients may be autoimmune-mediated. However, the target epitope(s) remain unknown.

Methods Human D2R mutants modified in their extracellular domains were subcloned, and we analysed the region bound by 35 anti-D2R antibody-positive patient sera using quantitative flow cytometry on live transfected cells.

Results No anti-D2R antibody-positive patient sera bound to the three extracellular loops, but all patient sera (35/35) targeted the extracellular N-terminus. Overall, patient antibody binding was dependent on two main regions encompassing amino acids 20 to 29 , and 23 to 37 . Residues 20 to 29 contributed to the majority of binding (77\%, 27/35), among which $26 \%(7 / 27)$ sera bound to amino acids R20, P21, and F22, 37\% (10/27) patients were dependent on residues at positions 26 and 29, that are different between humans and mice, and 30\% (8/27) sera required R20, P21, F22, N23, D26, and A29. Seven patient sera bound to the region 23 to 37 independently of D26 and A29, but most sera exhibited $\mathrm{N}$-glycosylation-independent epitope recognition at N23. Interestingly, no evident segregation of binding pattern according to patient clinical phenotype was observed.

Conclusions We report a major biological role for D2R extracellular N-terminus as a regulator of receptor surface availability, and as a major epitope targeted and impaired in brain autoimmunity. This knowledge could help the design of novel specific immune therapies tailored to improve patient outcome.

\section{NOVEL AUTOANTIBODIES AGAINST THE INTERFERON- RESPONSIVE MAJOR VAULT PROTEIN (MVP) IN SYSTEMIC LUPUS ERYTHMATOSUS}

${ }^{1} \mathrm{P}$ Budde*, 'HD Zucht, 'I Schulte-Pelkum, ${ }^{1} \mathrm{D}$ Wirtz, ${ }^{1} \mathrm{P}$ Rengers, ${ }^{2} \mathrm{~S}$ Vordenbäumen, ${ }^{2} \mathrm{M}$ Schneider, ${ }^{1} \mathrm{P}$ Schulz-Knappe. 'Protagen AG, Diagnostics, Dortmund, Germany; ${ }^{2}$ Heinrich-Heine University Düsseldorf, Rheumatology, Düsseldorf, Germany

\subsection{6/lupus-2017-000215.267}

Background and aims Autoantibody reactivity patterns are important disease and risk stratification marker in systemic lupus erythematosus (SLE). We have recently identified autoantibodies against the major vault protein (MVP) in SLE. Although the exact biological function of MVP is not well understood, MVP is an interesting autoantibody target, because it plays a pivotal role in virus-induced host response. MVP expression is induced by IFN $\gamma$ and induces upregulation of interferon (IFN) type I expression.

Methods Anti-MVP antibodies were discovered by high-content autoantibody profiling and validated in $>700$ SLE samples and autoimmune disease controls. To enable the development 
of smaller marker panels, we have developed anti-MVP into prototypic bead-based ELISA format.

Results Discovery and validation experiments using the NavigAID SLE array showed that anti-MVP antibodies occurred with frequencies of $15 \%-30 \%$ in three different SLE cohorts at a specificity of 97\%. Exploratory testing of multi-marker panels consisting of anti-MVP in combination with antidsDNA, anti-ribosomal $\mathrm{P}$ and anti-SmD yielded a $6 \%$ increase in sensitivity at $98 \%$ without loss of specificity. Multivariate data projection methods revealed that anti-MVP is detected in a subset of SLE patients with little overlap to established marker..A bead-based ELISA was developed for measuring anti-MVP antibodies and showed good correlation with Luminex data $(\mathrm{R}=0.88)$ indicating successful platform transfer.

Conclusions Anti-MVP autoantibodies represent a useful marker in SLE and, in combination with established markers, optimises the strategy for autoantibody testing. Furthermore, although more studies are needed, our findings suggest a previously undescribed linkage of type I IFN and autoantibody targets in SLE.

\section{AUTOANTIBODIES DICTATE CLINICAL MANIFESTATIONS IN PATIENTS WITH SYSTEMIC LUPUS ERYTHEMATOSUS}

${ }^{1,2}$ A Burlui* ${ }^{1,2}$ A Cardoneanu, ${ }^{1,2}$ E Rezus. ' Gr T Popa University of Medicine and Pharmacy, Rheumatology, lasi, Romania; ${ }^{2}$ Clinical Rehabilitation Hospital, Rheumatology, lasi, Romania

\subsection{6/lupus-2017-000215.268}

Background and aims Systemic Lupus Erythematosus (SLE) is known for its multifaceted clinical features and complex immune disturbance. Numerous studies have proven that certain autoantibodies are linked to specific clinical manifestations. However, the diversity of possible associations makes for the uniqueness of each case of SLE. The goal of our study was to analyse the link between clinical presentation and autoantibody titers in Romanian patients with SLE.

Methods We conducted an observational study of 48 adult patients with SLE hospitalised in the Rheumatology Department of the Clinical Rehabilitation Hospital. Venous blood samples were drawn to measure antinuclear antibody levels as well as anti-dsDNA, anti-ssDNA, anti-Sm, anti-U1RNP, antiSSA, anti-SSB and anti-nucleosome antibody titers (ELISA). Clinical presentation, biochemical tests, SLEDAI score values and urinalysis were extracted from patients ${ }^{6}$ charts. Patient characteristics were included in a database and analysed using IBM SPSS Statistics v20.

Results We found statistically significant correlations $(\mathrm{p}<0.05)$ between cutaneous manifestations and anti-Sm, anti-U1RNP, anti-SSA, anti-SSB and anti-nucleosome antibodies. Kidney involvement correlated with anti-Sm, anti-U1RNP and antinucleosome antibodies $(\mathrm{p}<0.05)$. Joint involvement was strongly associated with the presence of anti-U1RNP antibodies $(p=0.001)$. Haematological abnormalities were significantly correlated with anti-dsDNA, anti-U1RNP, anti-SSA and antiSSB antibodies $(p<0.05)$, while ESR and CRP levels were only associated with anti-U1RNP antibodies $(p=0.03)$. Furthermore, SLEDAI scores correlated with anti-dsDNA and antinucleosome antibody titers $(\mathrm{p}<0.05)$.

Conclusions Our data support the relationship between autoantibody titers, disease activity and severity of clinical changes in Romanian patients with systemic lupus erythematosus.

\section{SINGLE NUCLEOTIDE POLYMORPHISMS (SNPS) OF INTEGRIN-ALPHA-M (ITGAM) ARE ASSOCIATED WITH LUPUS NEPHRITIS (LN) IN AN ASIAN SYSTEMIC LUPUS ERYTHEMATOSUS (SLE) COHORT}

${ }^{1} \mathrm{M}$ Chan ${ }^{*},{ }^{1}$ WG Law, ${ }^{1} \mathrm{TY}$ Lian, ${ }^{1} \mathrm{KO}$ Kong, ${ }^{2} \mathrm{CY}$ Yu, ${ }^{3} \mathrm{YW}$ Song, ${ }^{1} \mathrm{HH}$ Chng, ${ }^{1} \mathrm{~B}$ Leung, ${ }^{4} \mathrm{~B}$ Tsao, ${ }^{1} \mathrm{HS}$ Howe. 'Tan Tock Seng Hospital, Rheumatology Allergy and Immunology, Singapore, Singapore; ${ }^{2}$ The Research Institute at Nationwide Children's Hospital, Centre for Molecular and Human Genetics, Columbus, USA; ${ }^{3}$ Seoul National University, Rheumatology, Seoul, Republic of Korea; ' University of California Los Angeles, Rheumatology, Los Angeles, USA

\subsection{6/lupus-2017-000215.269}

Background The Integrin-alpha-M (ITGAM) rs1143679 SNP has been associated with susceptibility to SLE and lupus nephritis (LN) in oriental Chinese and Thai populations. We previously found 13 ITGAM SNPs in linkage disequilibrium (LD) that were associated with susceptibility to SLE, but found no association with rs1143679.

Aim To determine associations of ITGAM SNPs with SLE subphenotypes and autoantibodies.

Methods We studied 248 patients fulfilling the 1997 ACR revised criteria for SLE. SLE-associated ITGAM SNP alleles were identified using custom-designed Immunochip arrays and gPLINK 1.062 software, with Bonferroni corrections for multiple comparisons. Associations of SLE-related ITGAM SNPs with SLE subphenotypes (malar or discoid rash, serositis, mouth ulcers, arthritis, haematological, renal or neurological involvement) and autoantibodies to dsDNA, Ro, RNP or Sm were determined with chi-square and Fisher's tests and logistic regression.

Results All 13 SLE susceptibility ITGAM SNPs as well as the uncommon rs1143679 SNP $(n=11)$ were associated with LN (Table 1). The strongest association was with rs2359661 $(p=0.002$, uncorrected). Subjects with these SNPs were less likely to have discoid rash. There was a trend towards an association with anti-Sm. Logistic regression models for 11 SNPs retained the factors $\mathrm{LN}$, discoid rash and anti-Sm, suggesting strong LD for these SNPs.

Conclusions This study demonstrated novel ITGAM SNP associations with LN and confirmed the association of rs1143679 with LN. Most associated SNPs were in the regulatory region of ITGAM bearing promoter/enhancer histone marks and have been associated with expression levels in several cell types, suggesting modulation of levels of ITGAM expression to impact these subphenotypes.

\section{ASSOCIATION OF TLR2 (23BP INS/DEL) POLYMORPHISM WITH SYSTEMIC LUPUS ERYTHEMATOSUS (SLE) AND P. FALCIPARUM MALARIA: A STUDY IN MALARIA ENDEMIC AREA OF ODISHA, INDIA}

${ }^{1}$ B Das*, ${ }^{2}$ R Tripathy, ${ }^{1} S$ Pattanaik, ${ }^{3}$ A Panda. ${ }^{1}$ S. C.B. Medical College, Medicine, Cuttack, India; ${ }^{2}$ S.C.B. Medical College, Biochemistry, Cuttack, India; ${ }^{3}$ Central University of Jharkhand, Centre for Life Sciences, Ranchi, India

\subsection{6/lupus-2017-000215.270}

Background and aims Human toll-like receptors (TLRs) participate in the innate response and signal the activation of adaptive immunity. TLRs play a vital role in sensing infection. A common $23 \mathrm{bp}$ insertion/deletion polymorphism at 5'UTR of TLR2 gene has been shown to affect TLR2 expression and plasma levels of pro-inflammatory molecules. We hypothesised 